\title{
Café com Saúde: Podcast como Ferramenta de Ensino nos Cursos de Saúde
}

\author{
Rayana M. C. M. Gomes1, Mariana P. Alencar1, Maria Julia M. N. Santos1, \\ Rosivan S. da Silva1, Júlio B. Messias1, Mônica S. Florêncio1 \\ 1Universidade de Pernambuco Campus Santo Amaro \\ raycaminha@gmail.com, pontesmarig9@gmail.com, majumns@hotmail.com, \\ julio.messias@upe.br, monica.simoes@upe.br
}

\begin{abstract}
The present article introduces the project Café com Saude, a pioneer pedagogical experience that uses the podcast as an intermediate for the democratization of the academic knowledge. Audio lessons were produced in the podcast format, being target to health undergraduate students. The lessons cover topics in histology, immunology, genetics, cellular biology, and other topics commonly present in theses courses' curriculum plans. The project was developed through literature review, elaboration of didactic scripts, recording, editing, marketing of the podcast and team meetings. The results were relevant, achieving efficiency to its target audience, reinforcing the use of podcasts as an educational tool.
\end{abstract}

Resumo. O presente artigo descreve o Projeto Café com Saúde, uma experiência pedagógica pioneira, que utiliza o podcast como vetor de democratização do conhecimento acadêmico. Foram desenvolvidas áudioaulas direcionadas a estudantes de nível superior dos cursos de saúde, abordando temas como histologia, imunologia, genética, biologia celular, dentre outros assuntos comuns à grade curricular destes cursos. O projeto contou com revisão bibliográfica, elaboração de roteiros didáticos, gravação, edição, divulgação e reuniões de equipe. Os resultados se mostraram relevantes, atingindo com eficiência o seu público alvo e fortalecendo o uso do podcast como ferramenta educacional.

\section{Introdução}

Café com Saúde é um projeto de extensão que usa a inovação tecnológica como ferramenta para tornar acessível temas dos cursos da Saúde e Ciências Biológicas, sob forma de podcast, aos diversos ouvintes, tendo alunos universitários como público alvo. Foram produzidas, entre março de 2018 e março de 2019, 10 audio-aulas nas quais discentes de cursos da área de saúde e um docente orientador fixo foram os anfitriões, conduzindo os episódios, sempre havendo no mínimo um profissional especialista no tema abordado como convidado. Os episódios foram publicados em plataformas virtuais de distribuição, como Spotify e Castbox.

Os temas abordados nos podcasts são relacionados à área de saúde, direcionados para alunos de graduação em Medicina, Ciências Biológicas, Enfermagem e Odontologia, inicialmente como um recurso educacional complementar às aulas de histologia, embriologia, genética, anatomia, biologia celular, imunologia e abordando patologias comuns nos serviços de atenção básica do SUS. 
O período das tecnologias já é o presente: todos os ramos da atividade humana estão sendo permeado por um crescente número de dispositivos, aplicativos e ferramentas relacionadas com as Tecnologias de Informação e Comunicação (TIC). E, nessa perspectiva, o ensino-aprendizagem também é influenciado e contemplado pelas ferramentas tecnológicas. A ideia de se desenvolver atividades e ferramentas interativas utilizando TIC surgiu da observação prática das dificuldades de aprendizagem em diversas disciplinas por parte de um número considerável de alunos, bem como da aproximação e familiaridade desses mesmos estudantes com as ferramentas e aplicativos de informática.

Segundo o método VAC, desenvolvido por Fernald e Keller e Orton-Gilingham (1920), o qual pressupõe que a aprendizagem ocorre por meio dos sentidos visual, auditivo e cinestésico; a maioria dos estudantes possui um estilo preponderante ou predileto para aprender os conteúdos das mais variadas disciplinas. Estudantes com preferência auditiva possuem diferenciada habilidade de reter conhecimentos captados através da linguagem sonora, sendo os principais, mas não os únicos, beneficiados com a ferramenta podcast.

Dessa forma, como citado por Vítor Diegues,(2010) o podcast possui inúmeros atributos, como a possibilidade de disponibilizar materiais didáticos na forma de aulas ou entrevistas em áudio, de ser acessado a qualquer momento sem limitação de localização e , também, disponibiliza informação para aqueles que dispõem de pouco tempo para a leitura e estudo, mas querem aprender.

A inovação do projeto consiste em usar as plataformas digitais, familiares aos discentes e acessíveis em smartphones, para transmitir conhecimento, fornecendo, dessa forma, aos alunos dos cursos de saúde, acesso à informação de qualidade produzida pela universidade.

Destaca-se que o projeto apresenta uma nova chance aos discentes de acessar a temática acadêmica e ainda os torna ativos do processo de aprendizagem, já que o conteúdo é acessado conforme desejam.

O objetivo do presente artigo é relatar a experiência do projeto Podcast Café com Saúde, descrever os resultados e revisar a literatura.

\section{Metodologia}

Trata-se de uma pesquisa-ação, com foco na aplicação de recursos tecnológicos no desenvolvimento de objetos educacionais direcionados aos acadêmicos dos Cursos de Medicina, Ciências Biológicas, Enfermagem e Odontologia.

A etapa descrita do projeto foi executada entre março de 2018 a março de 2019, utilizando o laboratório de informática e a rádio web da Reitoria da Universidade.

\subsection{Componentes curriculares}

O público alvo são os acadêmicos do ciclo básico dos cursos de Saúde e Ciências Biológicas. Houve a participação dos docentes das seguintes disciplinas: histologia, embriologia, genética, anatomia, imunologia, biologia celular, fisiologia e semiologia. Os referidos componentes curriculares foram desenvolvidos em áudios-aula de uma forma interdisciplinar, dotada de curiosidades e informações validadas pela literatura, estando relacionadas a conteúdos ministrados no ciclo básico dos cursos de saúde. 


\subsection{Revisão bibliográfica e contemporânea}

De forma inicial foi realizada uma pesquisa na literatura atual voltada ao uso do podcast como recurso educacional, bem como revisões bibliográficas para produção de cada episódio gravado. Dessa forma, as áudios-aula além de serem gravadas e validadas por docentes da área, estão atualizadas no meio científico da época. Estão também relacionadas a novidades contemporâneas veiculadas em jornais, legislação, recursos tecnológicos e outros aparatos de cunho construtivista na formação do discente.

Há mais de uma década, apontamentos científicos identificaram a revolução digital na saúde, associando às práticas de qualidade ao uso de computadores e incorporação das novas tecnologias [MENDES, TREVISAN e ÉVORA, 2000].

Esse avanço também é observado na área educacional, o que demanda uma melhor preparação para que esses profissionais sejam formados com capacidade para se adaptar e usufruir de toda a tecnologia da informação e comunicação [SILVA; MARQUES, 2011].

O avanço tecnológico fez surgir o conceito da Educomunicação, isto é, ações comunicativas no espaço educativo, com o objetivo de produzir e desenvolver ecossistemas comunicacionais em espaços educativos presenciais ou virtuais, ampliando o processo de aprendizagem. O termo Educomunicação foi cunhado pela primeira vez pelo filósofo da educação Mário Kaplun e seu conceito explicita uma junção da educação e da comunicação, firmando-se como um novo campo de intervenção, em que se busca ressignificar os movimentos comunicativos no âmbito da educação [SOARES et al,2002].

As tecnologias da informação e comunicação (TICs) estão ligadas a Educomunicação, sendo essenciais para a consolidação desse conceito. TICs são caracterizadas por processos de transmissão de dados através de dispositivos eletrônicos e recursos ópticos e têm seu maior produto na internet, considerada um poderoso instrumento de comunicação que diminui distâncias, interliga pesquisadores e permite que a informação produzida seja facilmente localizada em acervos das mais diversas áreas do conhecimento [COSTA et al., 2011]. Uma das áreas mais favorecidas com as TICs é a educacional. Seja na educação presencial ou a distância, o uso de TICs é potencializador do processo ensino-aprendizagem.

\subsection{Elaboração de roteiro didático}

Foram elaborados 10 roteiros didáticos, trazendo curiosidades e informações a respeito do assunto abordado no episódio. Através de pesquisa em conceituadas bibliotecas virtuais, como PubMed e Scielo, foram elaborados roteiros com uma introdução sobre o assunto, os principais tópicos a serem abordados e questionamentos comuns sobre o tema. Procurou-se abordar sobretudo as doenças relevantes para estudo pelos estudantes das diversas áreas da saúde e ciências biológicas, enfatizando sua epidemiologia, quadro clínico apresentado, diagnóstico e tratamento. Recursos digitais foram embutidos com finalidade de tornar mais lúdica a mídia a fim de otimizar a assimilação das informações emitidas pelos interlocutores na gravação, isso pôde ser realizado durante o processo da edição.

\subsection{Gravação, edição e divulgação}

Para as gravações foram firmadas parcerias com a rádio web da Universidade, a fïm de utilizar os recursos tecnológicos lá disponíveis. A gravação e edição foram realizadas diretamente no computador, usando como principal software livre de edição de áudio o Audacity. Foi adotada uma periodicidade de 15 dias a cada gravação, totalizando 2 Podcasts por mês. 
Foram ministradas capacitações sobre produção e edição de podcasts para alunos e professores envolvidos no projeto. Durante as edições foram direcionadas a dinâmica do episódio, a fluidez das conversas e os elementos de auxílio pedagógico.

Os arquivos em mp3 foram hospedados em uma plataforma virtual, com seu cadastro tendo sido devidamente registrado nas plataformas Castbox e Spotify. Assim, todos usuários de smartphones tem acesso ao nosso Podcast.

A divulgação é realizada por meio de perfis do projeto em redes sociais, como Instagram e Facebook, já que a maioria dos discentes participam dessas redes. São realizadas publicações periódicas que chamam a atenção do público-alvo com imagens ilustrativas, fotos das gravações e textos que resumem a temática abordada e convidam o usuário das redes a conhecer o projeto.

\subsection{Acompanhamento dos acessos}

A partir do portal de informação e do sistema de distribuição Spotify e Castbox, as estatísticas de acesso puderam ser obtidas. Dessa forma pode-se avaliar o número de acessos a cada episódio e, consequentemente, o impacto que gerado no público ouvinte.

\subsection{Reuniões da equipe}

Realizaram-se, durante a execução do projeto, reuniões semanais com os discentes e orientadora com o intuito de integrar a equipe, dividir tarefas, definir temas e professores convidados e, além disso, solucionar eventuais empecilhos à prática do projeto.

\section{Resultados}

Foram gravados e disponibilizados 10 episódios de podcasts nas plataformas virtuais Castbox e Spotify, com os seguintes títulos: "Saudações"; "Saúde da Mulher"; "Embriologia"; "Esquistossomose"; "HPV, o que não contaram pra você"; "Estresse e Ansiedade"; "A raiva"; "Câncer de Pele"; "O vírus HIV"; "Lúpus Eritematoso Sistêmico" (Tabela 1). 
Tabela 1. Título, duração e número de visualizações por episódio do podcast Café com Saúde.

\begin{tabular}{|l|c|c|}
\hline \multicolumn{1}{|c|}{ Título } & Duração do Episódio & Número de Visualizações \\
\hline 1. Saudações & 12 minutos e 21 segundos & 22 visualizações \\
\hline 2. Saúde da Mulher & 38 minutos e 49 segundos & 69 visualizações \\
\hline 3. Embriologia & 37 minutos e 04 segundos & 46 visualizações \\
\hline 4. Esquistossomose & 27 minutos e 04 segundos & 59 visualizações \\
\hline 5. HPV & 40 minutos e 02 segundos & 47 visualizações \\
\hline 6. Estresse e Ansiedade & 45 minutos e 22 segundos & 72 visualizações \\
\hline 7. A Raiva & 29 minutos e 46 segundos & 67 visualizações \\
\hline 8. Câncer de Pele & 26 minutos e 41 segundos & 67 visualizações \\
\hline 9. Vírus HIV & 41 minutos e 16 segundos & 151 visualizações \\
\hline 10. Lúpus Eritematoso Sistêmico & 54 minutos e 57 segundos & 131 visualizações \\
\hline
\end{tabular}

Foi obtido o total de 742 acessos, sendo 439 no Castbox e 303 no Spotify (Figura 1) entre março de 2018 e março de 2019.

\section{DISTRIBUIÇÃO DE ACESSOS POR PLATAFORMA}

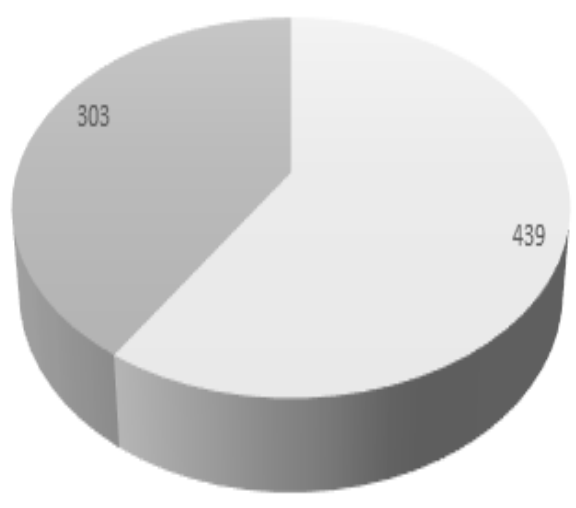

Castbox $=$ Spotify

\section{Gráfico 1. Distribuição de acessos entre as plataformas Spotify e Castbox.}

A plataforma Spotify forneceu a distribuição dos ouvintes do Café com Saúde por faixa etária (Figura 2), revelando que a maioria do público se encontra na faixa dos 18 aos 22 anos, idade da maior parte dos universitários. Também foi revelado que o público possui diversas nacionalidades, abrangendo ouvintes do Brasil, Alemanha, Itália e Estados Unidos (Figura 3).

Quanto à idade dos respondentes que ouvem podcast verificada em uma pesquisa de acordo com Felice (2018), a amostra apresentou um grupo com média entre os 20 e 29 anos $(56 \%)$. Eles são o publico ouvinte mais engajado da mídia, considerando que na última PodPesquisa (2014) a média de idade dos ouvintes foi de 25 anos. 


\section{DISTRIBUIÇÃO DE ACESSOS DO SPOTIFY POR IDADE}

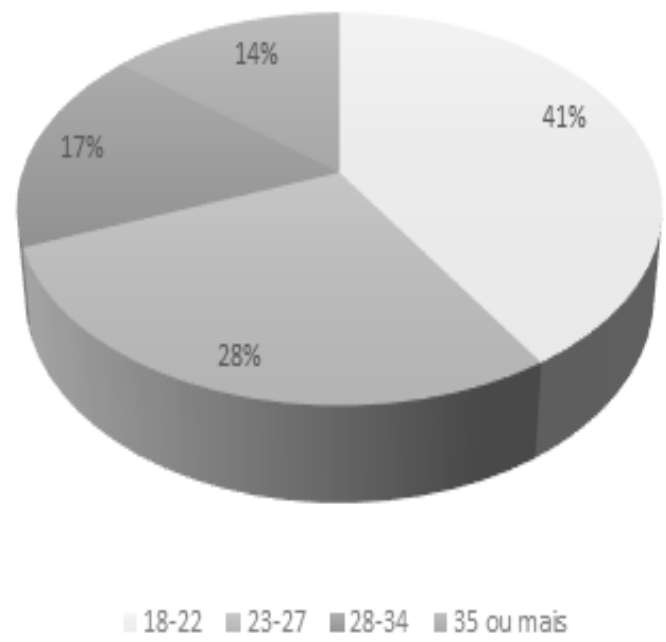

Figura 2. Distribuição de ouvintes do Spotify por faixa etária.

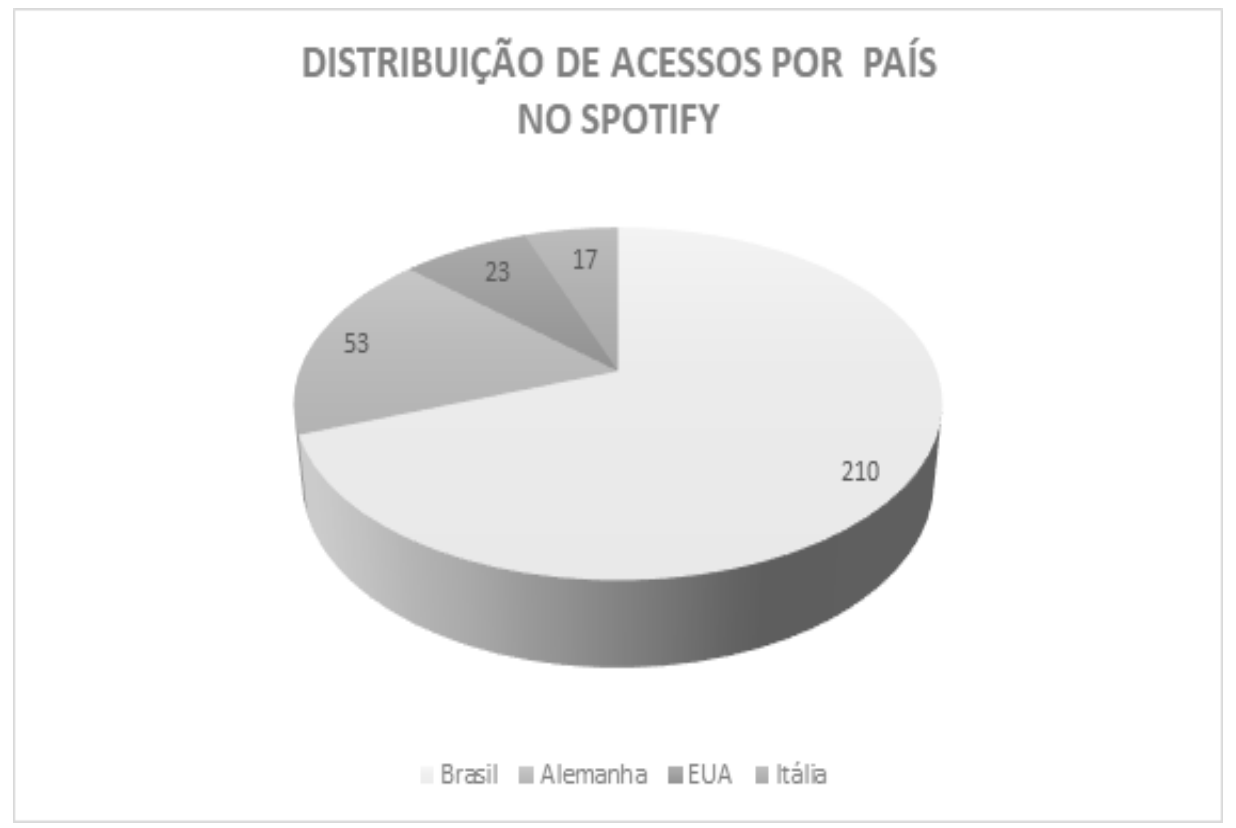

Figura 3. Distribuição de acessos no Spotify do podcast Café com Saúde entre países.

\section{4- Discussão}

Diante do avanço das tecnologias de informação e comunicação, a informática tornou-se uma ferramenta imprescindível para o desenvolvimento das áreas da saúde. Sua utilização impulsiona o rápido acesso às informações e ao conhecimento expandido no mundo todo, 
vencendo até mesmo distâncias geográficas, de modo a facilitar o desenvolvimento das mais variadas atividades [TANABE; KOBAYASHI, 2013].

Aliada ao processo de aprendizagem e atualização dos profissionais, a Internet, rede mundial de computadores, representa um recurso valioso para o profissional que busca novas informações. A Internet permite que barreiras físicas sejam superadas, possibilitando o desenvolvimento de cursos de educação a distância (EAD) com alto nível de interatividade, favorecendo o desenvolvimento de atividades tanto síncronas quanto assíncronas, suplantando outras formas de EAD como o rádio e a televisão [SANCHES; LOPES, 2008].

Nesse sentido, destaca-se a importância de ocupar o espaço da educação nas redes, com projetos como o Café com Saúde. Há a demanda de que as universidades brasileiras se alinhem com a tendência mundial de comunicação e disponibilização de conhecimento dos grandes centros universitários via plataformas digitais como forma complementar ao ensino tradicional.

Ainda sobre os recursos audiovisuais, tem-se como exemplo o Podcast, que consiste em arquivos recebidos por uma demanda de grupo ou assunto, podendo facilitar o processo de pesquisa e ensino destes. Por se tratar de arquivos com temas específicos, é possível o armazenamento das pesquisas e conteúdo. Apesar da produção crescente em diferentes sites da $W e b$, existem poucos que se voltam especificamente para o ensino [LEE et al, 2008 apud LEITE, 2012]. Contudo, tem-se algumas pesquisas que focam seu uso nas áreas educacionais [MOURA; CARVALHO, 2010; BOTTENTUIT JUNIOR; COUTINHO, 2008; LEITE, 2012; SANTOS; LEÃO, 2012]. Com isso, destaca-se o caráter inovador do uso de podcasts como meio complementar de ensino.

Segundo Silveira (2017) ocorre um grande diferencial entre os podcasts em relação a um broadcasting, por exemplo. Enquanto um broadcast é aquele programa de rádio transmitido de maneira simultânea, um podcast ocorre através de uma gravação que pode ser acessado pela internet a qualquer período e baixado pelos assinantes, sendo assim, os ouvintes possuem um perfil específico já que ouvir um podcast difere de ouvir uma rádio tradicional. Notadamente, o ouvinte é sempre o mesmo para todos os canais de podcasts existentes, havendo assim, uma disputa dos apresentadores em estar atualizando o canal com conteúdo de agrado ao público.

No contexto analisado por Salmon \& Fothergill (2007) foi analisado o uso de podcasts como meio complementar às atividades de ensino e de aprendizagem e como forma de motivar os alunos. Os resultados apontaram que os podcasts fornecem uma boa introdução aos recursos de aprendizagem disponibilizados e auxiliam a organização semanal das atividades de aprendizagem dos estudantes.

Os episódios de Podcast são arquivos de áudio, disponibilizados pelas plataformas Castbox e Spotify que podem ser encontradas gratuitamente por qualquer "smartphone", independente do sistema operacional. Com base nessas informações, é muito importante um projeto inovador como o Café com Saúde, que busca integrar o conhecimento da graduação, através da facilidade de atualização que a Internet oferece e a possibilidade de integrar profissionais e acadêmicos em um ambiente virtual. Através de podcasts, os acadêmicos envolvidos podem aprimorar habilidades e competências importantes para sua formação. Além disso, os usuários do projeto têm acesso a informações atualizadas e validadas por docentes, elaboradas com princípios científicos.

Através dos resultados obtidos, destaca-se que o projeto Café com Saúde atingiu seu público alvo de jovens universitários, já que a maioria dos ouvintes (69\%) possui 
entre 18 e 27 anos (Figura 2). Além disso, a plataforma Castbox concentrou 59\% dos acessos, sendo a mais utilizada para acessar o conteúdo (Figura 1).

O projeto teve alcance internacional, com 93 acessos, sendo 70 na Europa e 23 nos Estados Unidos (Figura 3). Podemos inferir que os brasileiros residentes nesses países se interessaram em buscar temas de saúde nas plataformas, corroborando para a nossa afirmação de que há carência desse tipo de conteúdo.

Analisando o número de visualizações dos episódios ao decorrer do projeto (Tabela 1), pode-se inferir que o aumento dos números representa uma alta aceitação e retenção de usuários do podcast Café com Saúde, o que serve como pequena amostra da crescente utilização do podcast como ferramenta educativa. Os resultados também revelam que houve uma satisfatória captação de novos ouvintes, demonstrando que o podcast gerou interesse em seu público alvo. Também foi possível constatar que a duração do episódio não tem clara relação com o número de visualizações.

\section{Considerações finais}

Num mundo globalizado onde o tempo é escasso, diferentes tecnologias vêm surgindo com o objetivo de facilitar a vida dos usuários em diversos aspectos, inclusive na educação. O podcast é uma dessas alternativas, sendo extremamente potente no processo de ensino e aprendizagem, tanto na modalidade a distância ou como no complemento ao ensino presencial. A flexibilidade espacial e temporal, a nível da gestão individual dos momentos e espaços de aprendizagem, é um dos contributos que o podcast vem trazer ao cenário educativo, barreiras se rompem e as fronteiras se aproximam, tornando o processo de aprendizado mais acessível, dinâmico e sem a necessidade de uma interrupção de outras atividades para que o conhecimento seja adquirido, como acontece por meios de educação tradicionais.

O projeto Café com Saúde gerou uma mobilização por parte de discentes e docentes para que, através de uma cooperação entre as áreas de saúde e tecnologia da informação, o aprendizado em saúde seja mais compreensível, direto, e amigável frente às novas tecnologias que permeiam praticamente todos os campos do conhecimento humano na atualidade.

Os resultados do projeto mostraram-se em consonância com o crescente espaço que o podcast vem ocupando como ferramenta digital educativa e de democratização do conhecimento, atraindo ouvintes por sua ampla variedade de benefícios e gerando interesse de alunos universitários de diversos cursos, como Medicina, Odontologia, Enfermagem, Ciências Biológicas.

\section{Referências}

COSTA, Priscila B. et al., 2011. Fluência digital e uso de ambientes virtuais: caracterização de alunos de enfermagem. Rev. esc. enferm. USP, São Paulo, v. 45, n. spe.

GROSSI, Manoela G. and KOBAYASHI, Rika M., 2013. “A construção de um ambiente virtual de aprendizagem para educação a distância: uma estrategia educativa em serviço.” Rev. esc. enferm. USP, São Paulo, v. 47.

FELICE, Giuliano B. B., 2018. "Como um Podcast é descoberto e escolhido? Investigando os consumidores dessa nova mídia." 38f. Trabalho de Conclusão de Curso - Universidade Federal de Uberlândia. 
LOPES, LEO, 2012 “Podcast: guia básico.” 1. ed. Nova Iguaçu: Marsupial Editora, 2015. $128 \mathrm{p}$.

MENDES, I.A.C. and TREVISAN, M.A. and ÉVORA, Y.D.M., 2000 "Comunicação e enfermagem: tendências e desafios para o próximo milênio." Esc. Anna Nery Rev.

MOMESSO, M.R., 2016 "Educar com podcasts e audiobooks." 1.ed. Porto Alegre: CirKula, 2016. 180 p. [e-Book].

PERES, H.H.C., MEIRA, K.C., LEITE, M.M.J., 2007 "Ensino de didática em enfermagem mediado pelo computador: avaliação discente.” Rev Esc Enferm USP.41 (2):271-8.

RESULTADO Geral de PodPesquisa 2014. ABPOD. Disponível em $<$ http:www.abpod.com.br/media/docs/Podpesquisa-2014.pdf $>$ Acesso em 7de ago. 20192

SANCHES, L.M.P., JENSEN, R. MONTEIRO, M.I., LOPES, M.H.B.M., 2011 "Informatics teaching in undergraduate nursing programs at Brazilian public institutions." Rev. Latino-Am. 19(6) 1385-90.

SANCHES, L.M.P.; LOPES, M.H.B.M., 2008 "Educação a distância sobre cardio versão e desfibrilação para enfermeiros." Rev Bras Enferm, Brasília 61(5): 583-8.

SILVA, I.S.A., MARQUES, I.R., 2011 "Conhecimento e barreiras na utilização dos recursos da Tecnologia da Informação e Comunicação por docentes de enfermagem." J. Health Inform. 3(1): 3-8.

SOARES, I. 2002. "Gestão Comunicativa e Educação: Caminhos da Educomunicação," in Comunicação \& Educação, n 23, pp. 16-125.

TANABE, Lyvia Pini; KOBAYASHI, Rika Miyahara, 2013“Perfil, competências e fluencia digital dos enfermeiros do Programa de Aprimoramento Profissional." Rev. esc. enferm. USP, São Paulo, v. 47, n.4. 